\title{
Linx
}

Revue des linguistes de l'université Paris X Nanterre

$12 \mid 2002$

"Comme la lettre dit la vie »

\section{Tant, étude d'un marqueur de transfert dans les textes épiques du moyen français}

Isabelle Weill

\section{(2) OpenEdition}

Journals

Édition électronique

URL : http://journals.openedition.org/linx/1320

DOI : $10.4000 /$ linx.1320

ISSN : 2118-9692

Éditeur

Presses universitaires de Paris Nanterre

\section{Édition imprimée}

Date de publication : 1 octobre 2002

Pagination : 276-280

ISSN : 0246-8743

\section{Référence électronique}

Isabelle Weill, «Tant, étude d'un marqueur de transfert dans les textes épiques du moyen français », Linx [En ligne], 12 | 2002, mis en ligne le 10 octobre 2012, consulté le 24 avril 2019. URL : http:// journals.openedition.org/linx/1320 ; DOI : 10.4000/linx.1320

Ce document a été généré automatiquement le 24 avril 2019

Département de Sciences du langage, Université Paris Ouest 


\title{
Tant, étude d'un marqueur de transfert dans les textes épiques du moyen français
}

\author{
Isabelle Weill
}

1 Michèle Perret, dans sa thèse consacrée à la localisation spatiale en moyen français (Perret, 1988: 5), a montré que dans une perspective diachronique il n'était pas évident de se pencher sur « des structures qui se sont maintenues dans une forme apparemment semblable du XI ${ }^{e}$ siècle à nos jours »; elle a noté qu'il était également difficile "d'éviter le réflexe des philologues cherchant à éviter toute solution de continuité entre le latin et l'ancien français"; elle a aussi constaté que la linguistique formelle étudiait de préférence les constituants nucléaires de la phrase mais que l'on pouvait pratiquer une autre linguistique en se consacrant aux éléments qui établissent des réseaux de relations anaphoriques et qui assurent par là la logique et la continuité des développements dans le discours et dans la narration. D'autres grands linguistes médiévistes se sont intéressés à des éléments de structuration des énoncés médiévaux, disparus en français moderne, mais je voudrais suivre les traces de ma collègue et amie nanterroise depuis plus d'un quart de siècle en présentant quelques éléments d'une recherche que j'espère pouvoir lui dédier un jour sous forme de livre achevé. Je m'intéresse au marqueur tant, un élément dont l'étude se trouve toujours dispersée dans de multiples chapitres des grammaires ; je voudrais m'élever contre les idées reçues et apporter ici quelques éléments pour tenter d'établir que le tant médiéval (dont il reste d'importantes traces en français classique, postclassique et même en français moderne), à l'opposé du tantus latin, ne note jamais l'intensif mais marque uniquement, avec un repérage anaphorique, la conséquence efficace ; on le trouve soit extraposé en tête de phrase, soit introduit juste avant l'élément repéré, soit dans un groupe prépositionnel (notre élément a pu sembler proche du pronom démonstratif $c e$ ) d'emploi anaphorique, soit placé, encore en extraposition, avant une conjonction (le que introducteur de complétive (après un verbe de type dire), que équivalent d'une locution en français moderne, comme en emploi temporel) qui conserve par ailleurs sa valeur habituelle dans la syntaxe médiévale. 
2 Ce marqueur (bien connu de tous les médiévistes pour les difficultés qu'engendre sa traduction en français moderne) fonctionne depuis le plus ancien français. Nous l'étudierons dans des textes épiques du $\mathrm{XIV}^{\mathrm{e}}$ et $\mathrm{XV}^{\mathrm{e}}$ siècles pour trois raisons : il s'agit évidemment d'une époque chère à Michèle Perret; en outre cette époque troublée marquée par des calamités accablantes (guerres, famines, pestes, pillages) et par la vision d'un monde descendant inexorablement vers la fin des temps, une apocalypse s'annonçant prochaine, voit se multiplier étonnamment les emplois de nos structures ${ }^{1}$ (on a encore bien mal étudié les rapports entre la langue et les états de pensée) ; enfin la chanson de geste, liée au genre historique, aime élaborer des mises en relief formulaires, dans un discours qui se veut stratégique pour décrire un univers décrit comme implacable et placé entre les mains d'une puissance supérieure.

3 Je vais, après avoir élaboré une hypothèse descriptive, étudier quelques variantes de cette structure de langue et de conscience remarquable par la régularité des mécanismes engendrés, dans des chansons du $\mathrm{XIV}^{\mathrm{e}}$ et $\mathrm{XV}^{\mathrm{e}}$ siècles dont certaines ne sont que récemment publiées: le Batard de Bouillon (la plus anciennement publiée), Florent et Octavien, Hughes Capet, L'histoire de Charlemagne (Fierabras), La belle Hélène de Constantinople, Galien le Restoré, Jourdain de Blayes en alexandrins, Le Roman de Guillaume d'Orange en prose. L'adverbe mar, étudié par B. Cerquiglini, indique une sorte de non fonctionnement, une discordance par rapport à une attente; or est pour M-L. Ollier un opérateur de rupture; si , étudié par C. Marchello-Nizia est un marqueur d'assertion. De même qu'en grec ancien la particule enclitique -te est un marqueur de généralisation, tant contient un signifié efficace de passage irréversible dans une dimension, un temps, un espace différent, voire un autre univers de pensée ; on pourrait penser au plateau de la balance qui bascule une fois le compte atteint ou de façon plus anachronique à un catalyseur en chimie, à une entrée validée pour ouvrir une nouvelle fenêtre en informatique ; c'est un outil qui, une fois les éléments précédents épinglés, s'enclenche et ancre la situation dans un nouvel état de relation au vrai; il canalise le possible (suivant peut-être le sens réductif de l'adverbe latin tantum au sens de seulement) et note l'écoulement inexorable du temps, le cours des destins liés à la norme de la condition humaine ou aux desseins particuliers de Dieu, la succession logique des événements qui va de la réussite normalement attendue à la catastrophe prévisible : tant note qu'on aboutit à un résultat stabilisé, voire rigidifié, échappant de façon irréfutable à toute discussion dans l'espace du texte. Ceci s'oppose évidemment à tout ce que représente pour nous le tantus latin marqué par une charge intensive certaine ; je citerai seulement en guise d'exemple un passage de Plaute mettant en scène un parasite heureux d'apporter à son hôte une très bonne nouvelle en pensant ainsi trouver toujours ouverte la porte de son nouvel obligé :

«Non ego nunc parasitus sum, se regum rex regalior

Tantus ventri commeatus meo adest in portu cilus. $»^{2}$

Ce que A. Ernout traduit : « je ne suis plus parasite, mais le roi des rois le plus royal, tant est superbe le convoi de vivres qui est entré dans le port à destination de mon ventre. » Notons que le latin médiéval évolue aussi et que tantum quod, au XIII ${ }^{\mathrm{e}}$ siècle, n'exprime plus que la simple conséquence.

5 Je commencerai par montrer comment l'interprétation traditionnelle de tant intensif en phrase exclamative me parait fausser le sens des textes. Je voudrais d'abord citer un texte de référence connu de tous, La Chanson de Roland, avec la traduction de G. Moignet, en regrettant de ne pouvoir en discuter avec mon ancien maitre, disparu au moment où je devenais la collègue de Michèle Perret à Nanterre; je pense que considérer tant comme 
un marqueur de consécution, de transfert fonctionnant de façon efficace permettra une autre approche de certains passages.

Dans la laisse XXXIV, Ganelon, en tant que messager de l'empereur, lance une sorte de défi à Marsile, lequel, furieux, menace aussitôt de le tuer ; Ganelon commence à tirer son épée du fourreau et adresse ces mots à cette dernière :

Si li ad dit : «Mult estes bele et clere!

Tant vus avrai en curt a rei portee!

Ja nel dirat de France li emperere

Que suls i moerge en l'estrange cuntree

Einz vos avrunt li meillor cumperee. (v.445-449)

Moignet traduit ainsi le vers 446 «je vous aurai tant portée en cour royale !» La phrase suivante n'est pas reliée à cet énoncé : «Jamais l'empereur de France ne dira que je sois mort seul en la terre étrangère avant que les meilleurs ne vous aient payée cher $! »$. On sait bien que la structure avec préposition $a$ dans « curt a rei » note l'indétermination; on devrait interpréter la phrase de façon itérative puisque de toutes les façons le verbe porter n'est pas susceptible de varier en gradation d'intensité ; mais Ganelon n'a pas passé sa vie à aller de cour de roi en cour de roi. Ganelon, le jour où il sent sa fin prochaine, ne peut donc que regretter d'avoir longuement, de jour en jour, porté noblement son épée à une cour qui ne peut être que celle de Charles. Ceci paraît étrange car il nommerait l'empereur à ce moment-là au lieu de le nommer au vers suivant. En outre le jour de leur mort, les guerriers se vantent des exploits accomplis avec leur épée, Roland rappelle à Olivier qu'ils ont "portée » (le même verbe est employé) Durandal et Hauteclère dans divers lieux et combats (v.1460-1467) et on ne voit pas pourquoi Ganelon, dont personne ne nie les qualités de courage, se réjouirait à sa mort, devant des étrangers, de souvenirs de vie de cour dont l'histoire n'a par ailleurs gardé aucune trace. L'ensemble paraît aberrant et je propose de supprimer le point d'exclamation et de lier le vers 446 au vers 447 par une parataxe ; Ganelon fait alors allusion, dans une formule de menace pleine de sous-entendus (la personne qui menace n'envisage que le résultat final, la mise à exécution de la menace étant escamotée comme s'il fallait effacer, nier toute responsabilité dans un événement qui se déroulerait en quelque sorte de lui-même), à la façon très anormale (mais terriblement efficace grâce au marqueur de bon fonctionnement) dont il va manipuler, utiliser son épée, à la cour d'un roi, celle où il se trouve, chez Marsile, roi qu'il méprise au point de refuser d'accorder à son nom la moindre importance; le futur antérieur indique que l'énonciateur, Ganelon, se place fictivement à un moment du temps qui suit sa mort à venir et on peut comprendre en enchaînant les deux phrases comme s'il y avait «tant que » et en glosant librement: "Voici ce que l'on dira de moi après ma mort : je vous aurai portée à la cour d'un certain roi en sorte que jamais l'empereur ne puisse dire que je sois mort seul en cette terre étrangère avant que les meilleurs hommes de cette cour n'aient payé au prix fort l'utilisation radicale et irrépressible que je vais faire de vous. " Le transfert entre les deux états successifs envisagés est présenté comme totalement accompli même si cette « réussite » est très amère pour Ganelon ; un piège, un gouffre sans échappatoire possible s'ouvre pour Marsile et ses meilleurs guerriers, ce qui les amène évidemment à tous réfléchir et à vouloir retenir l'ardeur de leur roi.

8 On peut citer aussi le passage où Ganelon tente de détourner Charles de se porter au secours de son neveu: 
Asez savez le grant orgoil Rolant;

Co est merveille que Dieu le soefret tant.

Ja prist il Noples seinz le vostre comant ; (v.1773-1775)

Moignet traduit : « Vous connaissez bien le grand orgueil de Roland; il est surprenant que Dieu le tolère si longtemps. Il a déjà pris Noples sans votre commandement. » Le verbe «souffrir » admet une variation en degré et en durée mais avec cette ponctuation qui interdit l'enchaînement entre les vers 1174 et 1775, on ne peut que s'étonner de voir que Ganelon présente Roland comme un ennemi de Dieu dont il faudra bien que le créateur un jour ou l'autre se débarrasse; il ne serait pas adroit pour le traître d'éveiller ainsi la colère de Charlemagne; mais si nous restituons, en supprimant toute ponctuation, une construction en parataxe, on peut interpréter : «il est surprenant que Dieu le tolère en sorte qu'il a pu prendre Noples sans votre commandement ». Ganelon devient alors habile en comptant la prise de Noples, dont le souvenir n'est sûrement pas agréable à l'empereur, parmi les petits péchés que Dieu peut pardonner au neveu de Charles en lui conservant sa protection.

D'autres vers beaucoup plus ambigus annoncent le passage d'un tant marqueur de consécution à un tant qui retournera à l'intensif en français moderne; il s'agit par exemple du passage dont il a été question plus haut, dans lequel Roland et Olivier font l'éloge de leurs épées :

En tant lius les avum nos portees!

Tantes batailles en avum afinees!

Male chançun n'en deit estre cantee.

Moignet traduit : « Nous les avons portées en tant de lieux! Nous avons achevé grâce à elles tant de batailles! Il ne faut pas qu'on chante d'elles une mauvaise chanson. »

Mon interprétation (qui change la valeur d'emploi modal de devoir) permet de supprimer toute crainte et toute inquiétude (sentiments bien mal venus avant une bataille chez des gens de cette trempe!) du discours de Roland: « nous les avons portées en des lieux tels et nous avons achevé des batailles grâce à elles de sorte qu'il n'y a pas de risque qu'on chante d'elles une mauvaise chanson ». Tant est marqué là par sa valeur de transfert et note l'entrée dans un univers de discours qui n'est plus celui de l'énonciateur (lequel sait qu'il va mourir) mais qui le dépasse infiniment puisque le marqueur le présente ici comme échappant à toute discussion et à tout risque de calomnie ; nous connaissons bien, dans tous les textes, cet emploi dans les relatives faisant l'éloge d'un personnage connu de tous ; si Olivier nous parle de « la fille au roi Hugues, qui tant est belle (Galien Restoré, p. 27), il ne veut pas par là marquer son admiration personnelle (on trouve dans ce cas moult, employé aussi pour les personnages qui sont connus du seul locuteur) mais indiquer que cette dame a été transférée dans un univers qui n'est plus celui du locuteur, un univers où sa beauté relève désormais du domaine de l'indiscutable et de l'infrangible ; ce type de relative emphatique, placé dans le deuxième hémistiche du vers est bien connu et sert à signaler que les héros sont passés dans l'espace légendaire de l'épopée ; relevons quelques-uns de ces éléments caractérisant, dans Hugues Capet, le héros «qui tant ot de renom » (v.1852), « qui tant est combatant » (v.1793)... C'est au fond le même emploi que l'on retrouve dans les formules épiques «la veïssiez tant...» qui réfèrent à l'espace intertextuel de la chanson de geste en son entier. On comprend en tout cas que ces tournures répétitives ont pu induire à penser que notre marqueur notait l'intensité.

Après avoir montré les valeurs sémantiques de notre marqueur, nous allons confirmer son emploi consécutif après des verbes tout aussi inattendus que repérés depuis 
longtemps : je parlerai d'abord des verbes de mouvement (chevauchier, errer, esploitier, se haster, faire (remplaçant normalement tous les verbes précédents) tant que) notant un déplacement, dans des tournures paraissant impossibles à traduire en français correct. C. Marchello Nizia $(1985: 85,98)$ a étudié les formules «tant chevauchier que » dans divers textes du XIII ${ }^{e}$ siècle en notant que le lieu d'aboutissement semblait soumis au hasard; je pense que l'on peut remplacer le mot de hasard par celui de fatalité inexorable : en dépit du sens de "bon fonctionnement" que tend à assumer notre marqueur, le hasard ne disparaît pas pour autant totalement de nos textes mais prend une autre forme : soit le héros arrive où il le désire, soit il rejoint les gens qu'il cherchait en un temps qui dépasse toutes ses espérances, soit il tombe dans une embuscade inattendue; il trouve ainsi son destin dans une phrase semblant fonctionner d'elle-même et échapper totalement à la volonté du sujet grammatical du verbe. Il peut s'agir de voyages très longs mais aussi de déplacements très brefs, preuve que l'idée d'efforts intensifs et soutenus ne fait pas partie du programme. Notons, parmi les emplois les plus courants, dans Galien le Restoré, la façon dont Ganelon se rend chez Marsile : «Or s'en alla Gannes et sa gent devers le roi Marsille. Si chevaucha tant qu'il vint la ou il se tenoict. » (p. 54); le départ de Charles paraît aussi anodin dans la phrase qui suit: "Si chevaucha tant l'empereur qu'il passa les porz " (p. 56). Dans ce passage du Roman de Guillaume d'Orange (p. 242), on peut voir que le déplacement n'est pas marqué par la durée : «A l'aprouchier d'Orange se parti Archillant d'avecque le roi Thiébaut et tant chevaulcha qu'il vint a Orenge. » Ces emplois n'ont rien de rare, on les trouve aussi dans Galien le Restoré (p. 49) : «Si chevauchierent Galien et Girard tant par la cité qu'ilz arriverent droit au palais. » Le résultat peut totalement dépasser les attentes de Guillaume et de ses amis (p. 198), lesquels rencontrent par hasard ou plutôt par le jeu de leur bonne destinée les Sarrasins que le roi Desramé a envoyés en ambassade : «Et tant chevauchierent les Crestïens apres Guillaume, qui devant poignoit tant comme il pouoit courre, que ils adevancerent les Sarrasins a l'issue d'un val ouquel il s'estoient boutez... ». La traduction par « en sorte que » parait dans la plupart des cas la plus fiable.

14 La négation produit des effets notables sur notre marqueur ; on traduit habituellement, dans les phrases où un verbe de mouvement est complément d'un verbe notant un arrêt et frappé par une négation, tant que par jusqu'à ce que, ce qui est certes une facilité mais ce qui ne rend pas compte de la valeur de notre marqueur qui garde toujours la même valeur de transfert : la convergence entre l'élément de départ et l'élément d'arrivée est retardée (ce que note l'emploi du subjonctif dans la subordonnée) mais elle devra avoir forcément lieu (avec une forme de subjonctif composé marquant l'accompli) ou l'on voit qu'elle a lieu (avec une forme simple du subjonctif); ainsi dans Guillaume d'Orange (p. 364), lorsqu'Ysaac, le messager d'Orable, se dit « qu'il ne cesseroit de cheminer tant que il eust le sien seigneur Guillaume veu », il faut comprendre non pas « il ne cessera de cheminer jusqu'à ce qu'il ait vu Guillaume » mais « afin de faire en sorte que sa mission, rejoindre Guillaume, soit entièrement accomplie ». Je pense que ces tournures ont fait disparaître les formules d'assertion avec $s i$, lesquelles ont justement disparu à l'époque de nos textes (Marchello-Nizia, p. 57). On les trouve, hors de toute formule de serment, dans le courant de la narration, ainsi dans Galien Rethoré (p. 231) : « Aprés ces paroles s'entrecomanderent a Dieu, et Godebeuf ne voulut onques arester tant qu'il arivast au tref de Charlemaine. ». L'action retardée (on retrouve l'idée d'un fonctionnement impossible à arrêter une fois enclenché) peut très bien ne pas dépendre du sujet initial de la phrase et dans ce cas le groupe verbal de sens négatif alterne avec un verbe positif marquant une attente, un moment d'immobilité, afin (le but est proche de la conséquence) que l'action attendue 
puisse se produire, une fois déclenchée par notre marqueur ; ainsi dans Galien le Restoré (p. 64), le roi Pinart refuse d'attaquer Galien qu'il a trouvé endormi dans un pré: «Or n'aies paour, car tant que tu soies tout ton corps habillé, ne te vouldroie touchier pour tout l'or du monde.». Les paroles du roi sont reprises dans la narration : « Or actendit le roy Pinart tant que Galien fust armé.». Il parait évidemment impossible de traduire autrement que par avant que, jusqu'à ce que, toute entreprise de traduction a des limites...

On a pu aussi croire que tant que avait été remplacé par jusqu'à ce que; en fait il n'en est rien et on peut le prouver en comparant les sens de jusqu'a ce que et jusqu'a tant que, formule mais dans laquelle l'apparition (par une commutation qui change tout le sens du contexte) de notre marqueur produit des effets qui n'ont rien d'anodin. On trouve jusqu'a ce que, dans les phrases où comptent les actions (jamais sous forme négative) qui remplissent l'attente, la rupture, le moment d'arrêt dans la narration, l'action de la subordonnée sur laquelle il n'y a pas aucune prise possible n'attirant pas la focalisation. Dans Guillaume d'Orange, le roi revient en France après la prise de Narbonne (p. 19) : « Si s'en parti Charlemeine a itant et enmena ses homes de son hostel, jusqu'a ce qu'il eust le Rosne passé. Puis envoya Gerart, le noble duc de Vianne en son païs, Renier à Gennes... ». Le passage du Rhône marque une limite, un arrêt et non pas le point de départ d'une action. Les exemples de ces structures sont nombreux et s'opposent aux formules incluant tant comme celle que nous notons dans Galien Rethoré (p. 207): Galien jure de retrouver son père et « ...dit que jamais ne cessera de cheminer tant qu'il aura trouvé son pere Olivier mort ou vif.». Il va alors trouver son grand-père, l'empereur de Constantinople pour demander congé et lui déclare : « ...ne cesserai d'aler par mons, par vaulx, par bois et par buyssons jusques atant que j'auray trouvé mon pere Olivier. ». Jusques a tant s'emploie avec d'autres verbes que les verbes de mouvement mais des verbes toujours frappés par la négation; ainsi dans Jourdain de Blaye, le traitre Fromont veut tuer le petit Jourdain après ses parents (v.703-704) :

Mais point n'est pour ytant ses voloirs acomplis

Jusqu'a tant que Jourdain sera mors et ochis.

16 On voit que la visée de jusqu'à ce que n'est pas du tout la même et tant que disparaitra en même temps que jusqu'a tant que pour noter les changements d'espace et de situation.

Sans verbe de mouvement notre marqueur note le transfert dans un autre type d'univers représenté comme achevé et stabilisé. Il sert donc à noter l'écoulement normal du temps qui se déroule évidemment de lui-même hors de toute intervention humaine et sans point de retour possible. Dans Jourdain de Blaye (v.125-126), Jourdain et son épouse enceinte se réjouissent

Ensy sont demenet le plus de la journee

Tant que li jours fali et revint l'avespree.

Dans Galien le Restoré (p. 35) Olivier et la belle Jacqueline oublient l'heure et se laissent surprendre par le jour : «Si s'embrasserent l'un l'autre jusques a tant qu'il fust grant jour, tant que Olivier s'eveilla... ». Les mêmes problèmes délicats mettent le jeune Hugues Capet dans une fâcheuse situation pour avoir aimé la fille d'un chevalier en Hainaut (v.184-186) :

La enama le fille d'un chevalier creeus

Tant qu'a privé fasoient lez sollas natureus.

A leur privé n'estoient de ce fait perecheus

Tant que celle fu grosse et sez ventres bocheus... 
19 Le père veut faire tuer ce héros qui n'est encore qu'un personnage de séduction et le jeune Hugues ne doit son salut qu'à une fuite rapide.

20 La vie humaine s'écoule comme le temps qui passe, tout commence évidemment par les naissances (Jourdain de Blayes, v.131-132) :

Se li aida tant Dieux et la Vierge loee

21 L'enfant du couple, le petit Jourdain, est confié à Renier et à l'épouse de ce dernier qui ont un enfant du même âge (v.298-299) et les enfants inéluctablement grandissent :

Et tant les eut Renier et le dame gardé

Que cascuns ot .IIII. ans, molt sont sage et sené

Et de l'escole apris, duit et endoctriné

Sy tres parfaitement qu'il ont latin parlé...

Cette structure sert aussi à noter les changements dans le monde certains désirés, d'autres non désirés mais tous aussi logiquement prévisibles que radicaux. Dans Hughes Capet, le héros constate que l'argent lui a filé entre les doigts (v.109-110) :

J'ay noblement hanté, s'ay donné maint biau don,

Tant que j'ay retenu le mains en ma parchon...

On retrouve fréquemment cette idée de compte et notre structure note le transfert dans un monde comptabilisé : dans Jourdain de Blaye, le roi Kalefrin rassemble ses troupes (v.11149-1150) :

Tant assamblerent gens au juste retraitier

C'on les peüst esmer a plus de.C.millier.

24 Ce comptage a pu également favoriser le passage à un tant intensif, étant donné qu'on n'éprouve le besoin de faire les comptes que dans le cas de quantités importantes.

Notre marqueur est aussi utilisé pour noter les réussites complètes. Les suites tant querir que trover sont fréquentes dans les textes depuis le XIII ${ }^{\mathrm{e}}$ siècle. Dans Jourdain de Blayes, Renier, protégé par un ange en prison, ne dépérit en aucune façon, ce qui provoque la colère du traître Fromont (v.1444-1445) :

Tant a mengiet Renier en le moie prison

Qu'il est sains et haitiez comme en le mer poissons !

Comprendre notre marqueur comme un intensif serait évidemment fâcheux dans ce contexte. Le personnage d'Hugues Capet paraît lui aussi un protégé de la fortune (v.76-77) :

...Et se fu de Fortune en le fin conjoïs

Tant que par hardement, par sens et par avis,

Fu roy de douche Franche, le nobile paîs..

On peut traduire alors certaines de ces structures par tant et si bien que, un subordonnant qui commence à apparaître à cette époque sous la forme tant et si que....

Mais les folies humaines aboutissent aussi à des catastrophes et notre marqueur note alors des réactions en chaînes cataclysmiques : l'herbe verte devient rouge du sang versé, le cours des rivières encombré de morts s'arrête de couler et déborde, ou peut encore être passé à pied sec... Ainsi dans Galien le Restoré (p. 34), un bourgeois vient prévenir le roi Hugues : «Sire, dist il au roy, trop sont de grant effort ces gens icy, et ont tué beaucoup de nos gens, tant que le sang en court par les rues, et Dieu leur peut bien aidier s'ilz ont droit et que nous aions tort. ».

29 Ici tant que correspond au moderne si bien que, notant le haut degré de conformité par rapport à une attente (cf Hybertie 1996 : 79-80). 


\section{On crie tant Noël que le fieste est trouvee, \\ Et sy puet estre a l'iaue le buire tant portee Qu'en le fin il se brise...} le montre ce « proverbe » pris dans Jourdain de Blayes (v.19392-19394) :

Dans cet état de langue, dès que la cruche va à l'eau, son destin fait qu'elle est entrée dans un monde ou, que ce soit au bout d'une journée ou que ce soit au bout de nombre d'années, tôt ou tard, inéluctablement elle se brisera. En français moderne (Hybertie 1996 : 77) « le constituant adverbial marque l'itération et fait dépendre l'avènement de la conséquence de l'itération ».

Nous n'avons abordé ici que les structures qui ont totalement disparu en français contemporain. J'espère continuer cette recherche dans le même esprit que Michèle Perret en écrivant la longue histoire de tant, tant en ancien français qu'en français moderne.

\section{BIBLIOGRAPHIE}

\section{Etudes}

CERQUIGLINI, B. (1981) La Parole médiévale, Paris : Les Editions de Minuit.

HYBERTIE, C. (1996) La conséquence en français, Paris : OPHRYS.

MARCHELLO-NIZIA, C. (1985) Dire le vrai : l'adverbe 'si' en français médiéval, Genève : Droz.

OLLIER, M.-L. (1995) « Or, opérateur de rupture, » in LINX, 32, 13-31.

PERRET, M. (1988) Le Signe et la mention : adverbes embrayeurs « ci », "ça », «la », « iluec » en moyen

français, Genève : Droz.

PERRET, M. (1995) « Histoire, nomination, référence », in LINX, 32, 173-188.

\section{Textes}

BANYON J., L'Histoire de Charlemagne, éd. par H.-E. Keller, Genève, Droz, 1992.

Le Batard de Bouillon, éd par R. F. Cook, Genève, Droz, 1972.

La belle Hélène de Constantinople, éd. par C. Roussel, Genève, Droz, 1995.

Florent et Octavien, éd. par N. Laborderie, Paris, Champion, 1991, 2 vols.

Galien le Restoré en prose, éd. par H.-E. Keller, Paris, Champion, 1998.

Hugues Capet, éd par N. Laborderie, Paris, Champion, 1997.

Jourdain de Blayes en alexandrins, éd. par T. Matsumura, Genève, Droz, 1999, 2 vols.

La Chanson de Roland, éd. par G. Moignet, Paris, Bordas, 1989.

Le Roman de Guillaume d'Orange, éd. par M. Tyssens, tome 1, Paris, Champion, 2000. 


\section{NOTES}

1. On compte dans le $\$ 3$ du chapitre XXX du Roman de Guillaume d'Orange (les références sont fournies dans la bibliographie), paragraphe qui fait vingt-cinq lignes, huit emplois de tant.

2. Plaute, Les Captifs, éd par A. Ernout, Paris, Budé, 1970, vv.825-826.

\section{AUTEUR}

\section{ISABELLE WEILL}

Université Paris 10 - Nanterre 